\title{
1 Sex-specific transgenerational plasticity I: Maternal and paternal effects on sons and
}

\section{2 daughters}

4 Jennifer K Hellmann ${ }^{1 *}$, Syed Abbas Bukhari ${ }^{1}$, Jack Deno ${ }^{1}$, Alison M Bell ${ }^{1,2,3}$

$6 \quad{ }^{1}$ Department of Evolution, Ecology and Behavior, School of Integrative Biology, University of

7 Illinois Urbana-Champaign, Urbana, Illinois, USA, 61801

$8 \quad{ }^{2}$ Carl R. Woese Institute for Genomic Biology, University of Illinois Urbana-Champaign,

9 Urbana, Illinois, USA, 61801

$10{ }^{3}$ Program in Ecology, Evolution and Conservation, University of Illinois Urbana-Champaign,

11 Urbana, Illinois, USA, 61801

12

13 "Corresponding author: Jennifer Hellmann, 505 S Goodwin Ave, Urbana IL 61801,

14 hellmann@illinois.edu 


\section{Abstract}

1. Transgenerational plasticity (TGP) or parental effects - when parental environments alter the phenotype of future generations - can influence how organisms cope with environmental change. An intriguing, underexplored possibility is that sex -of both the parent and the offspring - plays an important role in driving the evolution of transgenerational plasticity in both adaptive and nonadaptive ways.

2. Here, we evaluate the potential for sex-specific parental effects in a freshwater population of threespined sticklebacks (Gasterosteus aculeatus) by independently and jointly manipulating maternal and paternal experiences and separately evaluating their phenotypic effects in sons versus daughters. We tested the adaptive hypothesis that daughters are more responsive to cues from their mother, while sons are more responsive to cues from their father.

3. We exposed mothers, fathers, or both parents to visual cues of predation risk and measured offspring antipredator traits and brain gene expression.

4. Predator-exposed fathers produced sons that were more risk-prone, while predatorexposed mothers produced more anxious sons and daughters. Further, maternal and paternal effects on offspring survival were nonadditive: offspring with a predatorexposed father, but not two predator-exposed parents, had lower survival against live predators. There were also strong sex-specific effects on brain gene expression: exposing mothers versus fathers to predation risk activated different transcriptional profiles in their offspring, and sons and daughters strongly differed in the ways in which their brain gene expression profiles were influenced by parental experience.

5. We found little evidence to support the hypothesis that offspring prioritize their same-sex parent's experience. Parental effects varied with both the sex of the parent and the offspring in complicated and nonadditive ways. Failing to account for these sex-specific patterns (e.g., by pooling sons and daughters) would have underestimated the magnitude of parental effects. Altogether, these results draw attention to the potential for sex to influence patterns of TGP and raise new questions about the interface between transgenerational plasticity and sex-specific selective pressures, sexual conflict, and sexual selection. 
bioRxiv preprint doi: https://doi.org/10.1101/763862; this version posted June 17, 2020. The copyright holder for this preprint (which was not certified by peer review) is the author/funder, who has granted bioRxiv a license to display the preprint in perpetuity. It is made available under aCC-BY-NC-ND 4.0 International license.

45 Key words: maternal effect, paternal effect, Gasterosteus aculeatus, phenotypic plasticity,

46 intergenerational plasticity, nongenetic inheritance, predation, stress 


\section{Introduction}

Sex differences in life-histories (e.g. reproductive lifespan, mortality rate) or reproductive tactics can favor different optimal phenotypes in males and females (Andersson 1994). Although a shared genetic basis can constrain phenotypic differences between the sexes (Lande 1980;

51 Reeve \& Fairbairn 2001), epigenetic changes can overcome this constraint and allow males and

52 females to respond differently to the same environmental condition (within-generational

53 plasticity). Potentially adaptive sex-specific patterns of within-generational plasticity have been

54 documented in diverse taxa (Stillwell et al. 2010; Ceballos \& Valenzuela 2011; Xu et al. 2014;

55 Meuthen et al. 2018); for example, in cichlids, predation risk experienced early in life influenced

56 phenotypic development in males, but not females, possibly because males are more vulnerable

57 to predation (Meuthen et al. 2018).

While less explored, there also is evidence for sex-specific transgenerational plasticity

59 (TGP; also referred to as intergenerational plasticity or environmental parental effects);

60 specifically, the sex of the parent and/or the offspring can alter the ways in which environments

61 encountered by recent ancestors affect future generations. Empirical studies and theory to date

62 have primarily focused on maternal effects; however, the biological reality is that the

63 environment experienced by both mothers and fathers can affect future generations. For example,

64 there is growing evidence for paternal effects on ecologically-important traits, which can be

65 transmitted via paternal care as well as epigenetic changes to sperm (reviewed in (Crean \&

66 Bonduriansky 2014; Immler 2018)). Because males and females often experience different

67 environments once they reach reproductive age and have different means of transmitting

68 environmental cues to offspring (e.g. eggs versus sperm), the information transmitted by fathers

69 may not match the information encoded by mothers. Indeed, there is mounting empirical 
70 evidence that maternal versus paternal exposure to the same environmental condition can have

71 different effects on offspring (Bonduriansky \& Head 2007; Bonduriansky, Runagall-McNaull \&

72 Crean 2016; Gilad \& Scharf 2019). For example, previous studies have found both overlapping

73 and distinct gene expressions of maternal versus paternal experiences (Beemelmanns \& Roth

74 2016), which suggests that mothers and fathers can activate similar and different developmental

75 programs in their offspring depending on their experience. Further, the influence of maternal

76 environments might depend on paternal environments (or vice versa) (Mashoodh et al. 2012;

77 Mashoodh et al. 2018; Zirbel \& Alto 2018; Gilad \& Scharf 2019); for example, a recent study by

78 Lehto and Tinghitella (2020) found that stickleback females preferred duller males when either

79 their mother or father had encountered predation risk, but preferred brighter males when both

80 parents had experienced predation risk. Consequently, careful experimental studies that

81 independently and jointly manipulate maternal and paternal effects are needed to understand the

82 proximate and ultimate causes of similarities and differences between maternal and paternal

83 effects.

Parental effects also often depend on the sex of the offspring. For example, parental

85 environments can have opposing effects on the same trait in sons compared to daughters

86 (Mueller \& Bale 2007; Short et al. 2016; Braithwaite et al. 2017) or can influence different traits

87 in sons versus daughters (Schulz et al. 2011; Metzger \& Schulte 2016). For example, previous

88 studies have found divergent gene expression in sons and daughters in response to maternal

89 experiences (Metzger \& Schulte 2016; Constantinof et al. 2019), which suggests that maternal

90 experience activates different developmental programs in sons and daughters. Because the vast

91 majority of studies that have compared parental effects on sons and daughters have focused on

92 maternal effects, rather than both maternal and paternal effects (but see (Priest, Mackowiak \& 
93 Promislow 2002; He et al. 2016; Emborski \& Mikheyev 2019; Wylde et al. 2019)), it is often

94 unclear if sex-specific offspring effects are driven by 1) differences in the magnitude of sons'

95 versus daughters' responses to parental environments (e.g., daughters are generally more

96 responsive to parental stress, whether mediated by the mother or the father) or 2) how offspring

97 attend to experiences of their same-sex versus opposite-sex parent. Offspring may attend to

98 experiences of their same-sex parent (Bouwhuis, Vedder \& Becker 2015; Schroeder et al. 2015),

99 perhaps because sex differences in life history strategies or dispersal result in daughters being

100 more likely to encounter the environments experienced by their mothers, and sons being more

101 likely to encounter their fathers' environmental pressures. In order to evaluate the possibility that

102 offspring selectively prioritize experiences of one parent over the other, it is necessary to

103 compare maternal and paternal effects on both sons and daughters.

104 Sex-specific TGP might have important adaptive implications if it can resolve

105 evolutionary conflicts that occur when selection favors different phenotypes in males and

106 females (Bonduriansky \& Day 2008). Mothers and fathers may selectively alter the phenotypes

107 of their sons and daughters in response to the environment with a greater degree of precision than

108 genetic inheritance and in ways that may match the distinct life-history strategies of males and

109 females. Alternatively, sexual conflict could result in complex nonadaptive sex-dependent

110 patterns, especially when sexual selection is strong (Burke, Nakagawa \& Bonduriansky 2019).

111 Here, we evaluate the potential for sex-specific TGP in threespined sticklebacks (Gasterosteus

112 aculeatus). Male and female sticklebacks are sexually dimorphic in several respects, including in

113 habitat use (Reimchen 1980), diet (Reimchen \& Nosil 2001), parasite load (Reimchen \& Nosil

114 2001), and morphology (Reimchen, Steeves \& Bergstrom 2016), with these differences emerging

115 during early adulthood (Reimchen 1980; Reimchen \& Nosil 2001). Sexual selection favors a 
116 variety of male-specific reproductive traits that can increase males' vulnerability to predation

117 risk (Candolin 1998; Johnson \& Candolin 2017): male sticklebacks develop bright nuptial

118 coloration, engage in conspicuous territory defense and courtship behavior, and are the sole

119 providers of paternal care that is necessary for offspring survival (Bell \& Foster 1994). These sex

120 differences in behavior and life history often expose males and females to different predation

121 regimes (Reimchen \& Nosil 2004), likely altering the environment experienced by mothers

122 versus fathers and the optimal phenotype for daughters versus sons in response to predation risk.

123 We test the adaptive hypothesis that sex differences in life history strategies cause

124 offspring to attend to cues from their same-sex parent: we predicted that daughters would attend

125 to maternal cues and sons to paternal cues (i.e. a detectable interaction between maternal/paternal

126 treatment, and offspring sex). To test this hypothesis, we exposed adult male and female

127 sticklebacks to simulated predation risk prior to fertilization and used a fully factorial design to

128 generate offspring of control (unexposed) parents, offspring of predator-exposed mothers,

129 offspring of predator-exposed fathers, and offspring of predator-exposed mothers and fathers

130 (Figure 1). Because predation risk varies in both space and time, it is likely that there is a mix of

131 reproductively mature males and females who either have or have not recently experienced

132 predation risk within many natural populations. We reared sons and daughters under 'control'

133 conditions (i.e. in the absence of predation risk) and evaluated traits relevant to predator defense.

134 We then evaluated offspring brain gene expression patterns to assess whether maternal

135 experience with predation risk activates different developmental programs in offspring than

136 paternal experiences, and whether the experience of one parent (e.g. fathers) activates a

137 particular developmental program in one offspring sex but not the other (e.g. in sons but not

138 daughters). 


\section{Methods}

141 Housing conditions. Adult, sexually-mature, freshwater threespined sticklebacks were collected

142 from Putah Creek (CA, USA) in June-September 2016. This population has prickly sculpin

143 (Cottus asper), which preys primarily on stickleback eggs, fry, and juveniles. To simulate natural

144 conditions on the breeding grounds, where males defend nesting territories while females shoal,

145 we used different procedures for exposing mothers and fathers to predation risk. Females were

146 housed in six groups of $n=10$ fish per tank to mimic shoaling conditions in the wild. To simulate

147 predation risk, we used a clay model sculpin $(21 \mathrm{~cm}$ long) to chase females for 90 seconds each

148 day; unexposed treatment tanks were left undisturbed (similar to Dellinger et al. (2018)). Gravid

149 females were removed from tanks and stripped of their eggs for in-vitro fertilization; this

150 occurred the day after the last exposure for predator-exposed females. Control females were in

151 the tank for the equivalent period of time and removed at the same time of day as the predator-

152 exposed females to generate split clutches. Mothers were chased between 16-44 days, as mothers

153 took a variable amount of time to become gravid (see Supplementary Material for additional

154 analyses about the length of maternal exposure).

155 Males were housed singly to build nests. Once their nest was completed, predator-

156 exposed males were chased by a model sculpin for $30 \mathrm{sec}$ every other day for 11 days; control

157 males were left undisturbed. A separate experiment confirmed that the results reported below

158 were not produced when fathers were chased with a net (Chen et al. 2020), suggesting that

159 changes in offspring traits are specific to predation risk and not a byproduct of, for instance,

160 differences in activity levels due to chasing. The day after the last exposure, males were

161 euthanized to obtain sperm for in-vitro fertilization. While female sticklebacks produce clutches 
162 of eggs repeatedly throughout the breeding season, stickleback males produce sperm in the

163 beginning of the breeding season (Borg 1982); thus, paternal experiences mediated via sperm in

164 this experiment are likely due to modifications to mature sperm. We used a short stressor to

165 avoid reducing sperm quality or fertilization rates by exposing males to a stressor while

166 developing sperm and to avoid potential habituation to predation risk (Dellinger et al. 2018). By

167 beginning the treatment when males were transferred to a nesting arena, we sought to mimic the

168 change in predator regime that males may encounter as they move into a different habitat to nest.

While females were exposed to risk in groups, males were exposed to risk singly.

170 Because this regimen resulted in higher per capita risk for males than for females, males were

171 exposed for a shorter period of time than females. Although mothers and fathers were exposed to

172 risk differently, previous studies in this population suggest that exposure to predation risk for

173 individuals who are isolated versus in groups have largely similar consequences: another

174 experiment comparing post-fertilization paternal cues of predation risk with early life cues of

175 predation risk found largely overlapping effects of paternal and personal experience with

176 predation risk, despite the fact that fathers were exposed singly (with only one exposure) and

177 offspring were exposed in family groups repeatedly for a week (Stein, Bukhari \& Bell 2018).

178 F1 offspring were generated using a split clutch design, resulting in: 1) offspring of

179 unexposed fathers and mothers (n=11 half-clutches), 2) offspring of exposed fathers and

180 unexposed mothers ( $\mathrm{n}=11$ half-clutches), 3 ) offspring of unexposed fathers and exposed mothers

181 ( $\mathrm{n}=10$ half-clutches), and 4) offspring of exposed fathers and mothers ( $\mathrm{n}=10$ half-clutches). By

182 artificially fertilizing the eggs and incubating the embryos using an air bubbler, we controlled for

183 possible pre-fertilization effects mediated by interactions between mothers and fathers

184 (Mashoodh et al. 2012; McGhee et al. 2015), post-fertilization effects mediated by paternal care 
185 (Stein \& Bell 2014), and the possibility that predator-exposed parents might be less likely to

186 successfully mate or parent offspring. Separate groups of offspring were used for each assay

187 described below (Figure 1; see Supplementary Material for more detailed methods).

190 (mean: $20.6 \mathrm{~mm}$ standard length $\pm 2.1 \mathrm{~mm} \mathrm{s.d}$ ), groups of $\mathrm{n}=4$ offspring (one from each parental

191 treatment) were exposed to a live sculpin predator. One day prior to the predation assay, fish

192 were weighed, measured, marked, and individually transferred to a 250ml opaque glass beaker

193 containing $100 \mathrm{~mL}$ of water, with opaque sides to isolate the fish. We measured opercular beats

19430 seconds after transferring to the beaker as a proxy for acute stress (Bell, Henderson \&

195 Huntingford 2010) and 30 minutes after transferring to understand response to prolonged stress

196 (n=100 fish per parental treatment group). At the end of thirty minutes, all four fish were moved

197 to the same holding tank until the predation trial the following day. For the predation trial,

198 sticklebacks were simultaneously transferred into the sculpin's tank ( $\mathrm{n}=4$ different sculpin, each

199 used once per day); the trial ended two minutes after the first fish was captured by the sculpin.

$200 \quad 14 / 100$ trials did not result in any successful captures and were excluded from further analysis of

201 survival data. We euthanized the survivors of the predation assays and used a section of muscle

202 tissue to sex a large portion of the survivors per the methods of Peichel et al. (2004). We used

203 generalized linear mixed models (GLMM) with a binomial distribution (R package lme4 (Bates

204 et al. 2015)) to analyze differences in survival during the predation assay. We included fixed

205 effects of maternal treatment, paternal treatment, and standard length, with random effects of

206 maternal identity, paternal identity, sculpin identity, test group, and experimental day, to account

207 for potential improvement in sculpin performance over time. Because we found evidence of 
heteroskedasticity in our opercular beat data, we used MCMC generalized linear mixed models

209 (R package MCMCglmm (Hadfield 2010)) with a weak prior on the variance $(\mathrm{V}=1, \mathrm{nu}=0.002)$ to

210 analyze stress-induced respiration (breaths/minute). We ran models for 200,000 iterations, with a

211 burn-in of 3000 iterations, thin $=3$, and Gaussian distributions (and used these same parameters

212 all MCMC models below). We included fixed effects of maternal treatment, paternal treatment,

213 time period (30s or 30min), and standard length, as well as random effects of individual identity

214 nested within both maternal and paternal identity. We removed one extremely low outlier in the

215 opercular beat dataset. For all models, here and below, we tested for potential interactions

216 between maternal treatment, paternal treatment, and offspring sex during our model selection

217 process, and removed all not statistically significant interactions from final models.

219 Measuring risk taking behavior. When offspring were 4.5 months, we measured behavior in an

220 open field before and after a simulated predator attack (as in Bensky et al. (2017)). Individuals

221 were placed in an opaque refuge in the center of a circular arena $(150 \mathrm{~cm}$ diameter) divided into

222 eight peripheral sections with a circular section in the middle. After a three minute acclimation

223 period, we removed the plug from the refuge, allowed fish to emerge, and then measured the

224 number of different (exploration) and total (activity) sections visited for three minutes after

225 emergence. We then simulated a sculpin predator attack; this attack elicited freezing behaviour

226 from the fish and we measured the latency to resume movement after the simulated attack. Once

227 the individual resumed movement, we again measured the number of different and total sections

228 visited for three minutes. We weighed and measured the fish, euthanized it via decapitation, and

229 preserved the body in ethanol for identification of sex (Peichel et al. 2004). We assayed $n=118$

230 fish: $\mathrm{n}=12$ females and $\mathrm{n}=18$ males with control parents, $\mathrm{n}=15$ females and $\mathrm{n}=16$ males with 
231 predator-exposed fathers, $n=13$ females and $n=14$ males with predator-exposed mothers, and

$232 \mathrm{n}=11$ females and $\mathrm{n}=19$ males with two predator-exposed parents.

233 We used principal components analysis (R package factoextra (Kassambara \& Mundt

234 2017)) to combine exploration and activity (Spearman rank correlation: $\rho=0.92, p<0.001$ ), using

235 data from both before and after the simulated predator attack (two data points per individual).

236 We extracted an eigenvalue of 1.77 that captured $88.4 \%$ of the variance in these two behaviors;

237 positive values indicate more active and exploratory individuals. To understand how parental

238 exposure to predation risk altered offspring activity/exploration, length, and body mass, we used

239 MCMC GLMMs with a Gaussian distribution; we used MCMC GLMMs with a Poisson

240 distribution to analyze offspring freezing behavior. All models include fixed effects of maternal

241 treatment, paternal treatment, and individual sex. For activity/exploration, we included additional

242 fixed effects of standard length, and observation period (before or after the simulated predator

243 attack) and random effects of ID nested within mother and father identity (separately) and

244 observer identity. For freezing behavior we included additional fixed effects of standard length

245 and random effects of mother identity, father identity, and observer identity. We added an

246 additional fixed effect of age (days since hatching) to the model testing length and an additional

247 fixed effect of length for the model testing mass, with random effects of maternal and paternal

248 identity for both models. We removed one outlier for the length dataset and one different outlier

249 for the mass dataset.

251 Measuring anxiety/cautiousness. Scototaxis (light/dark preference) protocols have been

252 developed to test anti-anxiety/cautious behavior in fish (Maximino et al. 2010). When offspring

253 were between 9-13 months old, offspring were gently caught with a cup from their home tank 
254 and placed in a clear cylinder (10.5cm diameter) in the center of a half-black, half-white tank

255 (51L x 28W x $19 \mathrm{H} \mathrm{cm}$, coated on the inside and outside with matte contact paper). After a 5-

256 minute acclimation period, we lifted the cylinder, and fish explored the tank for 15 minutes,

257 during which we measured the latency to enter the white section, total time in the white section,

258 and the number of times the fish moved between the black/white sections. After the 15-minute

259 testing period, we removed the fish from the tank, recorded mass and standard length, euthanized

260 the fish in an overdose of MS-222, and confirmed sex via dissection and examination of the

261 gonads. The orientation of the tank was rotated between trials, and water was completely

262 changed between each trial.

263 On average, fish spent less time in the white portion of the tank than the black portion

264 (mean \pm s.e.: $208.7 \pm 18.8 \mathrm{sec}$ out of a $900 \mathrm{sec}$ trial). For individuals that never entered the white

265 side of the tank ( $\mathrm{n}=30$ of 162 individuals), we recorded latency to enter as 900 seconds. Because

266 all three variables were highly correlated (Spearman rank correlation; latency vs. time in white:

$267 \rho=-0.59, p<0.001$; latency vs. movement between sides: $\rho=-0.61, p<0.001$; time in white vs.

268 movement between sides: $\rho=0.78, p<0.001)$, we used principal components analysis ( $R$ package

269 factoextra) to combine these behaviors into one principal component (eigenvalue 2.10, captured

$27070.1 \%$ of the variance in behaviors): positive values were a measure of increased anti-

271 anxiety/more cautious behavior with a higher latency to enter the white section, less total time in

272 the white section, and fewer instances of switching between the black and white sections. We

273 then used this principal component as the dependent variable in a MCMC GLMM with a

274 Gaussian distribution, with fixed effects of maternal treatment, paternal treatment, sex, standard

275 length, and day, to control for any potential season effects. We also included random effects of

276 maternal and paternal identity, as well as observer identity. We assayed $n=162$ fish: $n=23$ 
277 females and $n=15$ males with control parents, $n=22$ females and $n=17$ males with predator-

278 exposed fathers, $n=23$ females and $n=21$ males with predator-exposed mothers, and $n=24$

279 females and n=17 males with two predator-exposed parents.

281 Measuring brain gene expression. We dissected whole brains from 4.5 month juvenile offspring

282 We sampled one male and one female offspring per family from 5 families per treatment group,

283 which totaled to $n=10$ ( 5 males, 5 females) offspring per treatment group ( $\mathrm{n}=40$ total). We

284 extracted RNA using Macherey-Nagel NucleoSpin 96 kits and sent $n=39$ samples to the

285 Genomic Sequencing and Analysis Facility at UT Austin for TagSeq library preparation and

286 sequencing (one sample was of poor quality). To estimate differential expression, pairwise

287 comparisons between the experimental conditions (offspring with only a predator-exposed

288 mother, offspring with only a predator-exposed father, offspring of predator-exposed mothers

289 and fathers) relative to the control condition (offspring of unexposed parents) within each sex

290 were made using edgeR (Robinson, McCarthy \& Smyth 2010). To call differential expression,

291 we used a 'glm' approach and adjusted actual p-values via empirical FDR, where a null

292 distribution of $\mathrm{p}$-values was determined by permuting sample labels for 500 times for each tested

293 contrast and a false discovery rate was estimated (Storey \& Tibshirani 2003).

294 In a separate analysis, we used WGCNA (R package WGCNA (Langfelder \& Horvath

295 2008) to cluster genes into co-expressed gene modules (Zhang \& Horvath 2005) and to reduce

296 the dimensionality of the transcriptomic dataset, which allowed us to explore the potential for

297 interactive effects of maternal treatment, paternal treatment and offspring sex on modules of

298 genes with correlated expression patterns. To find modules associated with treatment effects, we

299 fitted a linear model (Kuznetsova, Brockhoff \& Christensen 2017) which blocked for clutch ID 
as random factor, along with main and interactive effects of sex, paternal treatment, and maternal treatment on module eigengenes. Eigengenes which were significantly associated $(\mathrm{p}<0.05)$ with

302 either the main or interactive effects of sex, paternal treatment, and maternal treatment were

303 retained. See supplementary material for additional methods.

Animal welfare note. All methods were approved by Institutional Animal Care and Use

306 Committee of University of Illinois Urbana-Champaign (protocol ID 15077), including the use 307 of live predators.

\section{Results}

310 Sons, but not daughters, of predator-exposed fathers were more active under risk

311 In the open field assay, offspring were less active/exploratory after the simulated predator

312 attack compared to before (principal component analysis: higher values indicate more active and

313 explorative individuals; Table 1), confirming that offspring behaviorally responded to the

314 predator attack. There was a statistically significant interaction between paternal treatment and

315 offspring sex on offspring activity/exploration (Table 1; Figure 2A). Specifically, sons of

316 predator-exposed fathers were more active/exploratory compared to sons of control fathers

317 (MCMC GLMM, 95\% CI in brackets here and below [-1.30, -0.20], $\mathrm{p}=0.01$ ), but there was not a

318 detectable effect of paternal treatment on female offspring $([-0.40,0.81], p=0.49)$. In other

319 words, paternal effects on activity/exploratory behavior were stronger in sons, which is

320 consistent with our hypothesis.

321 We did not detect any maternal or paternal effects on freezing behavior (Table 1) or

322 standard length or body mass (see Supplementary Table 1). However, while we found no overall 
323 differences between offspring of control and predator-exposed mothers in length, among

324 offspring that had a predator-exposed mother, longer maternal exposure to the predator resulted

325 in larger offspring at 4.5 months (Supplementary Results). The length of exposure did not

326 significantly alter any other measured offspring traits (see supplementary material), although

327 there may have been an effect on other traits that were not measured in this study. Standard

328 length, mass, and freezing behavior (Table 1, Supplementary Table 1) also did not vary between

329 male and female offspring, although larger fish were less active/exploratory (Table 1).

331 Both sons and daughters of predator-exposed mothers, but not fathers, were more cautious

334 between black and white areas) compared to offspring of control mothers (Table 1; Figure 2B).

335 However, we did not detect an effect of paternal treatment on offspring scototaxis behavior

336 (Table 1). Both female and smaller offspring showed more cautious behavior, and we found no

337 evidence of seasonal effects (Table 1). Consequently, rather than offspring attending to the

338 experiences of their same-sex parent in the scototaxis assay, we observed that both sons and

339 daughters responded to maternal experiences.

\section{2 mother was also exposed}

343 There was a statistically significant interaction between maternal and paternal treatment

344 on offspring survival in live predation assays (generalized linear mixed effect model: $\mathrm{Z}_{334}=-$

$3451.98,0.048)$. Specifically, offspring of predator-exposed fathers were more frequently captured 
346 by the predator compared to offspring of control parents (Tukey's HSD with parental treatment

347 as a 4-factor variable: $Z=2.71, p=0.03$ ), but this was not true for offspring of predator-exposed

348 mothers $(\mathrm{Z}=0.75, \mathrm{p}=0.88)$ or both a predator-exposed mother and father $(\mathrm{Z}=-0.81, \mathrm{p}=0.85$;

349 Figure 2C). These results suggest that there was a strong fitness cost of having a predator-

350 exposed father, but mothers seemed to mitigate those costs, perhaps by making their offspring

351 more cautious (see above). Survivors of the successful predation trials were heavily female

352 biased (93/148; Chi-squared: $\left.\chi^{2}=9.76, p=0.002\right)$, suggesting that males are generally more

353 vulnerable to predation risk. The sex-bias was not statistically different across treatment groups

$354\left(\chi^{2}=3.03, p=0.39\right)$; this suggests that paternal exposure influenced sons and daughters equally,

355 although we cannot conclude this definitively because we do not know the sex of the captured

356 fish. We found no effect of size on how frequently the stickleback were captured by the predator

$357 \quad\left(\mathrm{Z}_{334}=1.56,0.12\right)$.

358 We did not detect any maternal or paternal effects on stress-induced respiration (see

359 Supplementary Table 1). For the portion of offspring where sex was known, we did not detect

360 any interaction between offspring sex and paternal $([-17.67,14.83], \mathrm{p}=0.89)$ or maternal

361 treatment $([-23.89,8.77], \mathrm{p}=0.37)$, although males tended to have higher opercular beats than

362 females (main effect of sex [-1.48, 25.92], $\mathrm{p}=0.08$ ).

Distinct maternal and paternal effects on offspring brain gene expression

To evaluate whether predation risk experienced by mothers versus fathers has different

366 consequences for offspring development at the molecular level, we compared the baseline brain

367 gene expression profile of offspring of unexposed parents (control) to offspring with a predator-

368 exposed mother, a predator-exposed father, and two predator-exposed parents in male and female 
369 offspring ( $\mathrm{n}=39$ individuals). In terms of the number of genes, maternal and paternal effects on

370 brain gene expression were approximately equivalent in magnitude, and the genes were largely

371 nonoverlapping (Figure 3A,B): in sons, for example, 1028 genes were differentially expressed in

372 response to maternal experience with risk, 904 genes were differentially expressed in response to

373 paternal experience with risk while only 253 genes were shared between them (daughters show a

374 similar pattern, Figure 3A). This suggests that, in contrast to our prediction, the transcriptomes of

375 sons and daughters are not more responsive to the experiences of their same-sex parent.

376 Interestingly, there was also a large number of genes that were unique to the "both" condition,

377 i.e. between offspring of two predator-exposed parents versus the control; these differentially

378 expressed genes could reflect the ways in which maternal and paternal effects interact at the

379 molecular level.

380 Of the differentially expressed genes that were shared between the pairwise comparisons,

381 nearly all were concordantly regulated, for both sons and daughters (Figure 3A,B). This suggests

382 that, despite the large-scale differences in brain gene expression between offspring of predator-

383 exposed mothers and fathers, there is a core set of genes that is activated in offspring brains in

384 response to either maternal or paternal exposure to predation risk.

386 Maternal and paternal exposure to predation risk interacted with offspring sex to influence

387 offspring brain gene expression

388 WGCNA identified 23 clusters ("modules") of genes with coordinated expression

389 patterns in the dataset. The expression of eight of the 23 modules was affected by at least one of

390 the factors in the model: three modules were affected by maternal treatment, two were affected

391 by the two-way interaction between maternal and paternal treatment, and three were affected by 
392 the three-way interaction between paternal treatment, maternal treatment and offspring sex

393 (shown in Figure 3C). For example, the module "saddle brown" comprises 48 co-expressed

394 genes (largely enriched for developmental processes) whose expression was influenced by the

395 three-way interaction between maternal treatment, paternal treatment and offspring sex.

396 Specifically, daughters of a predator-exposed mother or father showed lower expression of genes

397 in this module compared to daughters of control parents or two predator-exposed parents (Figure

398 3C). For sons, on the other hand, the expression of genes in this module was more strongly

399 affected by maternal treatment. A similar pattern was observed in the yellow and cyan modules.

400 Overall these results demonstrate that at the molecular level, daughters and sons differ in the

401 extent to which they respond to predation risk that had been experienced by their mother, father

402 or by both parents. However, in contrast to our overall hypothesis, there was no evidence that

403 sons and daughters primarily attend to experiences of their same-sex parent at the molecular

404 level.

405

406 Discussion

407 Here, we report the results of a comprehensive comparison of maternal and paternal

408 effects on sons and daughters. We show that both the sex of the parent and the sex of the

409 offspring influence the ways in which offspring phenotypes are altered by parental experiences.

410 However, we found little evidence for the adaptive hypothesis that offspring would attend

411 primarily to cues from their same-sex parent. Instead, our results illustrate the complexities of

412 sex-specific parental effects. Maternal and paternal effects in response to the same environmental

413 factor were largely distinct: predator-exposed mothers produced more cautious offspring

414 (scototaxis), while predator-exposed fathers produced sons, but not daughters, that were more 
415 active under risk (open field assays). Further, the effects of paternal predation exposure were

416 moderated by maternal predation exposure: offspring of predator-exposed fathers, but not two

417 predator-exposed parents, had reduced survival against a live predator. Yehuda et al. (2014)

418 found this same interactive pattern previously in humans due to parental PTSD and originally

419 attributed it to potential changes in parenting behaviors. Our results demonstrate that these non-

420 additive interactions can be mediated in the absence of behavioral interactions between parents

421 and offspring. Instead, our brain gene expression profile results - that there are distinct

422 neurogenomic changes in offspring with two predator-exposed parents compared to offspring of

423 either a predator-exposed mother or father - are consistent with the hypothesis that non-additive

424 patterns may arise because offspring phenotypes are influenced by the interaction between

425 environmentally-induced epigenetic changes in eggs versus sperm.

426 Sex-specific patterns - where sons and daughters differ in their response to maternal and

427 paternal exposure to predation risk - emerged in our study well before offspring were

428 reproductively mature, during a period in their life when males and females are shoaling and

429 occupying similar habitats (Bell \& Foster 1994). In contrast to our prediction that offspring

430 would adaptively attend to the cues of their same-sex parent, these sex-specific patterns of

431 parental effects did not seem to emerge along a consistent male-female divide (e.g. sons attend to

432 their father and daughters attend to their mother); instead, sons and daughters were both altered

433 by paternal and maternal environments, but in different ways. This is consistent with Emborski

434 and Mikheyev (2019), who found that male offspring were influenced by maternal, but not

435 paternal, diet. These sex-specific effects may be adaptive for offspring, with differences

436 originating in early development to allow offspring to develop phenotypes that are better

437 matched to the different environments they will encounter later in life. For example, it is possible 
438 that increased activity under risk for sons, but not daughters, may be adaptive because high

439 variance in male reproductive success favors males that adopt high risk, high reward behaviors to

440 increase growth and access to resources under high predation pressure (Bell, Henderson \&

441 Huntingford 2010). Alternatively, these effects may not be adaptive, either resulting from

442 differences in sons and daughters in their susceptibility to parental stress (Bale 2011; Glover \&

443 Hill 2012) and/or reflecting sexual conflict and sexually antagonistic selection (Burke,

444 Nakagawa \& Bonduriansky 2019). Indeed, Burke, Nakagawa and Bonduriansky (2019) suggest

445 adaptive TGP may be unlikely to arise in systems with sex-specific selection because sex-

446 specific ecologies can result in mothers and fathers experiencing different environments and

447 therefore transmitting conflicting information to their offspring.

$448 \quad$ Our study shows that maternal and paternal predation exposure can have fitness

449 consequences for offspring (i.e., via survival) in the lab; work is needed in a more natural context

450 in the field to assess the fitness consequences of parental effects. For example, there are multiple

451 steps required to avoid predation (Lima \& Dill 1990; Guiden et al. 2019); while our data suggest

452 that offspring with predator-exposed fathers are poor at evading predators once they come into

453 contact with predators, parental experience with predation risk might alter the likelihood that

454 offspring initially avoid coming into contact with predators. Further, offspring of predator-

455 exposed fathers might face a trade-off between survival and reproduction, favoring high-risk,

456 high-reward strategies that reduce survival in high predation environments, but increase

457 reproductive success by ensuring that surviving individuals are in good breeding condition.

458 Indeed, sticklebacks do seem to face a trade-off in surviving predation and gaining the body size

459 necessary for successfully reproducing, with this trade-off being stronger in males compared to

460 females (Bell et al. 2011). 
Whether the fitness interests of mothers, fathers, and offspring align or conflict has

462 important implications for the evolution of sex-specific TGP (Burke, Nakagawa \& Bonduriansky

463 2019). When parents' and offspring fitness interests in the face of predation risk are aligned, sex-

464 specific plasticity may arise because mothers and fathers experience their environment in

465 different ways and/or because the same parental environment favors different phenotypes in sons

466 and daughters. However, sex-specific TGP may arise because mothers and fathers favor different

467 optimal offspring phenotypes (Saldivar et al. 2017), and/or sons and daughters have different

468 capacities to respond to or ignore information from fathers and mothers. If this is the case, TGP

469 may evolve at the interface between sexual conflict and parent-offspring conflict, with paternal

470 strategies, maternal strategies, and offspring counter-adaptations all ultimately dictating

471 offspring phenotypes. This may result in the evolution of mechanisms that allow mothers to

472 manipulate the ways in which fathers influence offspring (e.g. via cytoplasmic contributions

473 (Crean \& Bonduriansky 2014)) or fathers to manipulate the ways in which mothers influence

474 offspring (e.g. via ejaculate composition (Garcia-Gonzalez \& Dowling Damian 2015)).

475 Interactions between maternal effects, paternal effects, and offspring sex could be

476 mediated via a variety of proximate mechanisms. Distinct maternal and paternal effects could

477 reflect different proximate mechanisms that mediate the transmission of cues from mothers

478 versus fathers to offspring (e.g., egg hormones or mRNAs versus sperm small RNAs) as well as

479 the ways in which mothers and fathers were exposed to risk. Both distinct and interactive effects

480 could also be mediated by epigenetic mechanisms such as parent-of-origin effects (Kong et al.

481 2009; Lawson, Cheverud \& Wolf 2013) or interactions between maternal and paternal

482 contributions (e.g. egg cytoplasm altering the effect of sperm small RNAs) during early

483 development (Crean \& Bonduriansky 2014; Garcia-Gonzalez \& Dowling Damian 2015). 
Differences between sons and daughters in how they respond to parental information could be

485 mediated via trans-acting mechanisms (e.g., regulation of genes on non-sex chromosomes by

486 genes located on the sex chromosome (Metzger \& Schulte 2016)), sex-specific differences in

487 epigenetic mechanisms, or genomic imprinting (Bonduriansky \& Day 2008; Dunn \& Bale 2011).

488 Further, in bulls, Y-bearing and X-bearing spermatozoa have differentially expressed proteins,

489 suggesting a mechanism by which fathers can transmit different information to sons versus

490 daughters (Scott et al. 2018). Although mothers in many species can also transmit different

491 information to sons and daughters (e.g., via placental function and gene expression (Bale 2011;

492 Glover \& Hill 2012)), it is unclear if mothers can transmit different information to sons and

493 daughters in externally fertilizing species such as sticklebacks, in which mothers do not interact

494 with their offspring post-fertilization. Future work exploring these proximate mechanisms could

495 help explain the extent to which variation in parental effects is due to changes in the information

496 encoded by parents or changes in offspring responsiveness to parental information.

497 Because parents can differentially allocate based on their partner's phenotype or

498 environmental conditions experienced by their partner (Mashoodh et al. 2012; McGhee et al.

499 2015; Mashoodh et al. 2018), in most systems it is difficult to isolate the effects of direct

500 parental exposure to an environmental cue from environmental cues that parents indirectly detect

501 from their mate (e.g. predator-naïve fathers provide less care to offspring of predator-exposed

502 mothers) (Mashoodh et al. 2012; McGhee et al. 2015; Mashoodh et al. 2018). This makes it

503 difficult to understand whether paternal effects can be mediated via sperm alone, or to determine

504 the influence of paternal effects in isolation of maternal effects. In this experiment, we were able

505 to completely isolate paternal effects mediated via sperm because there was no opportunity for

506 parents to interact pre-fertilization or to influence offspring post-fertilization. Although our 
507 results suggest that distinct and interactive effects of maternal and paternal effects can be

508 mediated via selective changes to information encoded in eggs and sperm alone, a fascinating

509 direction for future work would be to consider how parental care and mate choice might

510 ameliorate or magnify the sex-specific effects observed here.

\section{Conclusions}

513 Transgenerational plasticity can allow environmental information to be delivered to

514 offspring earlier and with potentially lower costs to offspring than developmental plasticity (Bell

515 \& Hellmann 2019). TGP can potentially be fine-tuned to the precise environment that both

516 parents and offspring will encounter (Bonduriansky \& Day 2008), perhaps including the

517 different environments experienced by males and females because of sex differences in life

518 history and reproductive tactics. We found that paternal cues mediated via sperm seem to be just

519 as prominent in terms of their magnitude and prevalence as maternal cues mediated via eggs,

520 although differences in our exposure regime (due to stickleback biology) may have influenced

521 the relative strength of maternal versus paternal effects. Furthermore, we show that offspring

522 phenotypes varied depending on whether predation risk had been experienced by their mother or

523 their father, and a parent's experience with predation risk produced different phenotypes in their

524 sons compared to their daughters. However, these sex-specific patterns would have been masked

525 if we had combined cues coming from mothers and fathers (i.e. compared offspring of two

526 predator-exposed parents to a control) or failed to isolate effects emerging in sons versus

527 daughters. Consequently, theoretical and empirical work seeking to understand the evolution of

528 transgenerational plasticity would benefit from considering the conditions which influence sex-

529 specific patterns of transgenerational plasticity in both adaptive and nonadaptive ways. Further, 
530 given broad interest in understanding the consequences of transgenerational plasticity for future

531 generations and its potential to influence adaptive evolution, future work should consider how

532 sex-specific effects in the first generation may alter the ways in which intergenerational effects

533 persist for multiple generations in lineage-specific and/or sex-specific ways.

534

535 


\section{Acknowledgements}

537 Thank you to Eunice Chen, Erin Hsiao, Yangxue Ma, Liam Masse, and Christian

538 Zielinksi for help with data collection and to Sarah Donelan and the Bell lab for comments on

539 previous versions of this manuscript. This work was supported by the National Institutes of

540 Health award number 2R01GM082937-06A1 to Alison Bell and National Institutes of Health

541 NRSA fellowship F32GM121033 to Jennifer Hellmann. The authors have no conflicts of

542 interest.

\section{Author contributions}

$545 \mathrm{JKH}$ and AMB designed the study. JKH generated offspring, conducted survival assays,

546 collected opercular beat data, dissected brains and extracted RNA, and oversaw open field assays

547 and offspring sexing. JD conducted scototaxis assays. SAB conducted gene expression analyses.

$548 \mathrm{JKH}$ wrote the first draft of the manuscript and JKH/AMB edited the manuscript.

550 Data accessibility

551 All datasets (survival, respiration data, scototaxis, behavioral assays, lists of differentially

552 expressed genes, read counts per sample, WGCNA) will be made publicly available on Dryad

553 upon acceptance of this manuscript. 


\section{References}

Andersson, M. (1994) Sexual selection. Princeton University Press, New York.

Bale, T.L. (2011) Sex differences in prenatal epigenetic programing of stress pathways. Stress, 14, 348-356.

Bates, D., Mächler, M., Bolker, B. \& Walker, S. (2015) Fitting linear mixed-effects models using lme4. Journal of Statistical Software; Vol 1, Issue 1 (2015).

Beemelmanns, A. \& Roth, O. (2016) Biparental immune priming in the pipefish Syngnathus typhle. Zoology, 119, 262-272.

Bell, A.M., Dingemanse, N.J., Hankison, S.J., Langenhof, M.B. \& Rollins, K. (2011) Early exposure to nonlethal predation risk by size-selective predators increases somatic growth and decreases size at adulthood in three-spined sticklebacks. Journal of Evolutionary Biology, 24, 943-953.

Bell, A.M. \& Hellmann, J.K. (2019) An integrative framework for understanding the mechanisms and multigenerational consequences of transgenerational plasticity. Annual Review of Ecology, Evolution and Systematics, 50, 97-118.

Bell, A.M., Henderson, L. \& Huntingford, F.A. (2010) Behavioral and respiratory responses to stressors in multiple populations of three-spined sticklebacks that differ in predation pressure. Journal of Comparative Physiology B, 180, 211-220.

Bell, M.A. \& Foster, S.A. (1994) The evolutionary biology of the threespine stickleback. Oxford University Press, Oxford.

Bensky, M.K., Paitz, R., Pereira, L. \& Bell, A.M. (2017) Testing the predictions of coping styles theory in threespined sticklebacks. Behavioural Processes, 136, 1-10.

Bonduriansky, R. \& Day, T. (2008) Nongenetic inheritance and its evolutionary implications. Annual Review of Ecology, Evolution, and Systematics, 40, 103-125.

Bonduriansky, R. \& Head, M. (2007) Maternal and paternal condition effects on offspring phenotype in Telostylinus angusticollis (Diptera: Neriidae). Journal of Evolutionary Biology, 20, 2379-2388.

Bonduriansky, R., Runagall-McNaull, A. \& Crean, A.J. (2016) The nutritional geometry of parental effects: maternal and paternal macronutrient consumption and offspring phenotype in a neriid fly. Functional Ecology, 30, 1675-1686.

Borg, B. (1982) Seasonal effects of photoperiod and temperature on spermatogenesis and male secondary sexual characters in the three-spined stickleback, Gasterosteus aculeatus L. Canadian Journal of Zoology, 60, 3377-3386.

Bouwhuis, S., Vedder, O. \& Becker, P.H. (2015) Sex-specific pathways of parental age effects on offspring lifetime reproductive success in a long-lived seabird. Evolution, 69, 17601771.

Braithwaite, E.C., Murphy, S.E., Ramchandani, P.G. \& Hill, J. (2017) Associations between biological markers of prenatal stress and infant negative emotionality are specific to sex. Psychoneuroendocrinology, 86, 1-7.

Burke, N.W., Nakagawa, S. \& Bonduriansky, R. (2019) Sexual conflict explains diverse patterns of transgenerational plasticity. bioRxiv, 846287.

Candolin, U. (1998) Reproduction under predation risk and the trade-off between current and future reproduction in the threespine stickleback. Proceedings of the Royal Society B, 265, 1171. 
Ceballos, C.P. \& Valenzuela, N. (2011) The role of sex-specific plasticity in shaping sexual dimorphism in a long-lived vertebrate, the snapping turtle Chelydra serpentina. Evolutionary Biology, 38, 163.

Chen, E., Zielinski, C., Deno, J., Singh, R., Bell, A.M. \& Hellmann, J.K. (2020) The specificity of sperm-mediated paternal effects in threespined sticklebacks. bioRxiv, doi: https://doi.org/10.1101/2020.06.04.135152.

Constantinof, A., Moisiadis, V.G., Kostaki, A., Szyf, M. \& Matthews, S.G. (2019) Antenatal glucocorticoid exposure results in sex-specific and transgenerational changes in prefrontal cortex gene transcription that relate to behavioural outcomes. Scientific Reports, 9, 764.

Crean, A.J. \& Bonduriansky, R. (2014) What is a paternal effect? Trends in Ecology \& Evolution, 29, 554-559.

Dellinger, M., Zhang, W., Bell, A.M. \& Hellmann, J.K. (2018) Do male sticklebacks use visual and/or olfactory cues to assess a potential mate's history with predation risk? Animal Behaviour, 145, 151-159.

Dunn, G.A. \& Bale, T.L. (2011) Maternal high-fat diet effects on third-generation female body size via the paternal lineage. Endocrinology, 152, 2228-2236.

Emborski, C. \& Mikheyev, A., S. (2019) Ancestral diet transgenerationally influences offspring in a parent-of-origin and sex-specific manner. Philosophical Transactions of the Royal Society B: Biological Sciences, 374, 20180181.

Garcia-Gonzalez, F. \& Dowling Damian, K. (2015) Transgenerational effects of sexual interactions and sexual conflict: non-sires boost the fecundity of females in the following generation. Biology Letters, 11, 20150067.

Gilad, T. \& Scharf, I. (2019) Separation between maternal and paternal effects on offspring following exposure of adult red flour beetles to two stressors. Ecological Entomology, 44, 494-501.

Glover, V. \& Hill, J. (2012) Sex differences in the programming effects of prenatal stress on psychopathology and stress responses: An evolutionary perspective. Physiology \& Behavior, 106, 736-740.

Guiden, P.W., Bartel, S.L., Byer, N.W., Shipley, A.A. \& Orrock, J.L. (2019) Predator-prey interactions in the Anthropocene: reconciling multiple aspects of novelty. Trends in Ecology \& Evolution, 34, 616-627.

Hadfield, J.D. (2010) MCMC methods for multi-response generalized linear mixed models: the MCMCglmm R package. Journal of statistical software, 1, 1-22.

He, N., Kong, Q.-Q., Wang, J.-Z., Ning, S.-F., Miao, Y.-L., Yuan, H.-J., Gong, S., Cui, X.-Z., Li, C.-Y. \& Tan, J.-H. (2016) Parental life events cause behavioral difference among offspring: Adult pre-gestational restraint stress reduces anxiety across generations. Scientific Reports, 6, 39497.

Immler, S. (2018) The sperm factor: paternal impact beyond genes. Heredity, 121, 239-247.

Johnson, S. \& Candolin, U. (2017) Predation cost of a sexual signal in the threespine stickleback. Behavioral Ecology, 28, 1160-1165.

Kassambara, A. \& Mundt, M. (2017) factoextra: extract and visualize the results of multivariate data analyses. R package version 1.0.5. https://CRAN.R-project.org/package=factoextra.

Kong, A., Steinthorsdottir, V., Masson, G., et \& al (2009) Parental origin of sequence variants associated with complex diseases. Nature, 462, 868. 
643

644

Kuznetsova, A., Brockhoff, P. \& Christensen, R. (2017) lmerTest package: tests in linear mixed effects models. Journal of statistical software, 82, 1-26.

Lande, R. (1980) Sexual dimorphism, sexual selection, and adaptation in polygenic characters. Evolution, 34, 292-305.

Langfelder, P. \& Horvath, S. (2008) WGCNA: an R package for weighted correlation network analysis. BMC Bioinformatics, 9, 559.

Lawson, H.A., Cheverud, J.M. \& Wolf, J.B. (2013) Genomic imprinting and parent-of-origin effects on complex traits. Nature Reviews Genetics, 14, 609.

Lehto, W.R. \& Tinghitella, R.M. (2020) Predator-induced maternal and paternal effects independently alter sexual selection. Evolution, 74, 404-418.

Lima, S.L. \& Dill, L.M. (1990) Behavioral decisions made under the risk of predation: a review and prospectus. Canadian Journal of Zoology, 68, 619-640.

Mashoodh, R., Franks, B., Curley, J.P. \& Champagne, F.A. (2012) Paternal social enrichment effects on maternal behavior and offspring growth. Proceedings of the National Academy of Sciences, 109, 17232-17238.

Mashoodh, R., Habrylo, I.B., Gudsnuk, K.M., Pelle, G. \& Champagne, F.A. (2018) Maternal modulation of paternal effects on offspring development. Proceedings of the Royal Society B, 285, 20180118.

Maximino, C., Marques de Brito, T., Dias, C.A.G.d.M., Gouveia Jr, A. \& Morato, S. (2010) Scototaxis as anxiety-like behavior in fish. Nature Protocols, 5, 209.

McGhee, K.E., Feng, S., Leasure, S. \& Bell, A.M. (2015) A female's past experience with predators affects male courtship and the care her offspring will receive from their father. Proceedings of the Royal Society B, $\mathbf{2 8 2}$.

Metzger, D.C. \& Schulte, P.M. (2016) Maternal stress has divergent effects on gene expression patterns in the brains of male and female threespine stickleback. Proceedings of the Royal Society B, 283.

Meuthen, D., Baldauf, S.A., Bakker, T.C.M. \& Thünken, T. (2018) Neglected patterns of variation in phenotypic plasticity: age- and sex-specific antipredator plasticity in a cichlid fish. The American Naturalist, 191, 475-490.

Mueller, B.R. \& Bale, T.L. (2007) Early prenatal stress impact on coping strategies and learning performance is sex dependent. Physiology \& Behavior, 91, 55-65.

Peichel, C.L., Ross, J.A., Matson, C.K., Dickson, M., Grimwood, J., Schmutz, J., Myers, R.M., Mori, S., Schluter, D. \& Kingsley, D.M. (2004) The master sex-determination locus in threespine sticklebacks is on a nascent Y chromosome. Current Biology, 14, 1416-1424.

Priest, N.K., Mackowiak, B. \& Promislow, D.E.L. (2002) The role of parental age effects on the evolution of aging. Evolution, 56, 927-935.

Reeve, J.P. \& Fairbairn, D.J. (2001) Predicting the evolution of sexual size dimorphism. Journal of Evolutionary Biology, 14, 244-254.

Reimchen, T.E. (1980) Spine deficiency and polymorphism in a population of Gasterosteus aculeatus: an adaptation to predators? Canadian Journal of Zoology, 58, 1232-1244.

Reimchen, T.E. \& Nosil, P. (2001) Ecological causes of sex-biased parasitism in threespine stickleback. Biological Journal of the Linnean Society, 73, 51-63.

Reimchen, T.E. \& Nosil, P. (2004) Variable predation regimes predict the evolution of sexual dimorphism in a population of threespine stickleback. Evolution, 58, 1274-1281.

Reimchen, T.E., Steeves, D. \& Bergstrom, C.A. (2016) Sex matters for defence and trophic traits of threespine stickleback. Evolutionary Ecology Research, 17, 459-485. 
Robinson, M.D., McCarthy, D.J. \& Smyth, G.K. (2010) edgeR: a Bioconductor package for differential expression analysis of digital gene expression data. Bioinformatics, 26, 139140. effect on offspring size linked to parent-of-origin expression of an insulin-like growth factor. Ecology and Evolution, 7, 4465-4474.

Schroeder, J., Nakagawa, S., Rees, M., Mannarelli, M.-E. \& Burke, T. (2015) Reduced fitness in progeny from old parents in a natural population. Proceedings of the National Academy of Sciences, 112, 4021.

Schulz, K.M., Pearson, J.N., Neeley, E.W., Berger, R., Leonard, S., Adams, C.E. \& Stevens, K.E. (2011) Maternal stress during pregnancy causes sex-specific alterations in offspring memory performance, social interactions, indices of anxiety, and body mass. Physiology \& Behavior, 104, 340-347.

Scott, C., de Souza, F.F., Aristizabal, V.H.V., Hethrington, L., Krisp, C., Molloy, M., Baker, M.A. \& Dell' Aqua, J.A. (2018) Proteomic profile of sex-sorted bull sperm evaluated by SWATH-MS analysis. Animal Reproduction Science, 198, 121-128.

Short, A.K., Fennell, K.A., Perreau, V.M., Fox, A., O’Bryan, M.K., Kim, J.H., Bredy, T.W., Pang, T.Y. \& Hannan, A.J. (2016) Elevated paternal glucocorticoid exposure alters the small noncoding RNA profile in sperm and modifies anxiety and depressive phenotypes in the offspring. Translational Psychiatry, 6, e837.

Stein, L.R. \& Bell, A.M. (2014) Paternal programming in sticklebacks. Animal Behaviour, 95, 165-171.

Stein, L.R., Bukhari, S.A. \& Bell, A.M. (2018) Personal and transgenerational cues are nonadditive at the phenotypic and molecular level. Nature Ecology \& Evolution, 2, 13061311.

Stillwell, R.C., Blanckenhorn, W.U., Teder, T., Davidowitz, G. \& Fox, C.W. (2010) Sex differences in phenotypic plasticity affect variation in sexual size dimorphism in insects: from physiology to evolution. Annual Review of Entomology, 55, 227-245.

Storey, J.D. \& Tibshirani, R. (2003) Statistical significance for genomewide studies. Proceedings of the National Academy of Sciences, 100, 9440.

Wylde, Z., Spagopoulou, F., Hooper, A.K., Maklakov, A.A. \& Bonduriansky, R. (2019) Parental breeding age effects on descendants' longevity interact over 2 generations in matrilines and patrilines. PLOS Biology, 17, e3000556.

Xu, W., Zhang, J., Du, S., Dai, Q., Zhang, W., Luo, M. \& Zhao, B. (2014) Sex differences in alarm response and predation risk in the fresh water snail Pomacea canaliculata. Journal of Molluscan Studies, 80, 117-122.

Yehuda, R., Daskalakis, N.P., Lehrner, A., Desarnaud, F., Bader, H.N., Makotkine, I., Flory, J.D., Bierer, L.M. \& Meaney, M.J. (2014) Influences of maternal and paternal PTSD on epigenetic regulation of the glucocorticoid receptor gene in Holocaust survivor offspring. American Journal of Psychiatry, 171, 872-880.

Zhang, B. \& Horvath, S. (2005) A general framework for weighted gene co-expression network analysis. Statistical Applications in Genetics and Molecular Biology.

Zirbel, K.E. \& Alto, B.W. (2018) Maternal and paternal nutrition in a mosquito influences offspring life histories but not infection with an arbovirus. Ecosphere, 9, e02469. 
734 Table 1: Results of general linear mixed models (MCMCglmm) testing predictors of

735 exploration/activity (higher values indicate more active and exploratory individuals) and freezing

736 behavior in the open field assay, as well as anxiety-like behavior in the scototaxis assay. We

737 tested for potential interactions between maternal treatment, paternal treatment, and offspring

738 sex; we removed not statistically significant interaction terms.

\begin{tabular}{|l|c|c|c|}
\hline \multirow{2}{*}{} & \multicolumn{3}{|c|}{ Activity and exploration } \\
\cline { 2 - 4 } & Mean & $95 \% C I(L, U)$ & $P$ \\
\hline Observation period & -0.97 & $-1.24,-0.70$ & $<\mathbf{0 . 0 0 1}$ \\
\hline Maternal treatment & 0.14 & $-0.27,0.54$ & 0.48 \\
\hline Paternal treatment & -0.20 & $-0.81,0.42$ & 0.52 \\
\hline Offspring sex & -0.25 & $-0.70,0.22$ & 0.29 \\
\hline Standard length & -0.12 & $-0.18,-0.05$ & $<\mathbf{0 . 0 0 1}$ \\
\hline Paternal treatment $*^{*}$ sex & 0.91 & $0.25,1.54$ & $\mathbf{0 . 0 0 5}$ \\
\hline & \multicolumn{3}{|c|}{ Freezing behavior } \\
\hline & Mean & $95 \% C I(L, U)$ & $P$ \\
\hline Maternal treatment & 0.31 & $-0.15,0.80$ & 0.19 \\
\hline Paternal treatment & -0.17 & $-0.65,0.31$ & 0.47 \\
\hline Offspring sex & -0.36 & $-0.78,0.07$ & 0.09 \\
\hline Standard length & 0.06 & $-0.02,0.15$ & 0.14 \\
\hline & \multicolumn{3}{|c|}{ Anxiety-like behavior } \\
\hline & Mean & $95 \% C I(L, U)$ & $P$ \\
\hline Maternal treatment & 0.58 & $0.07,1.11$ & $\mathbf{0 . 0 3}$ \\
\hline Paternal treatment & -0.19 & $-0.70,0.30$ & 0.44 \\
\hline Offspring sex & -0.72 & $-1.27,-0.17$ & $\mathbf{0 . 0 1}$ \\
\hline Standard length & -0.05 & $-0.10,-0.005$ & $\mathbf{0 . 0 3}$ \\
\hline Experimental day & 0.004 & $-0.004,0.01$ & 0.33 \\
\hline
\end{tabular}




\section{Figures}

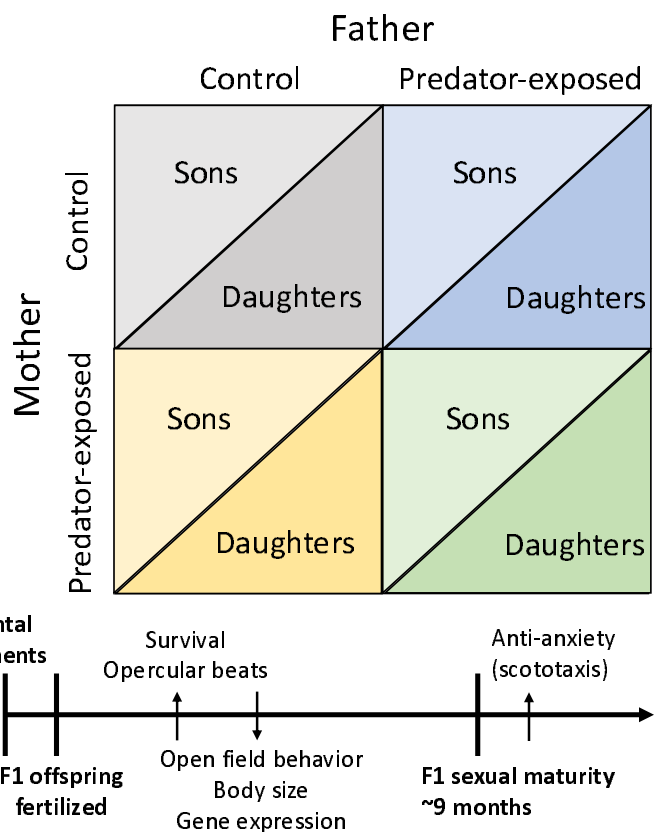

743 Figure 1: Experimental diagram of parental treatments with experimental timeline. Both male

744 and female F0s were either left undisturbed or exposed to predation risk prior to fertilization and

745 then crossed in a full-factorial, split-clutch design to generate F1 sons and daughters. On the

746 timeline, bolded words represent key events while non-bolded words represent experimental

747 measurements. Distinct sets of F1 offspring were used to measure 1) survival and opercular

748 beats, 2) open field behavior and body size, 3) gene expression, and 4) anti-anxiety behavior 749 (scototaxis). 

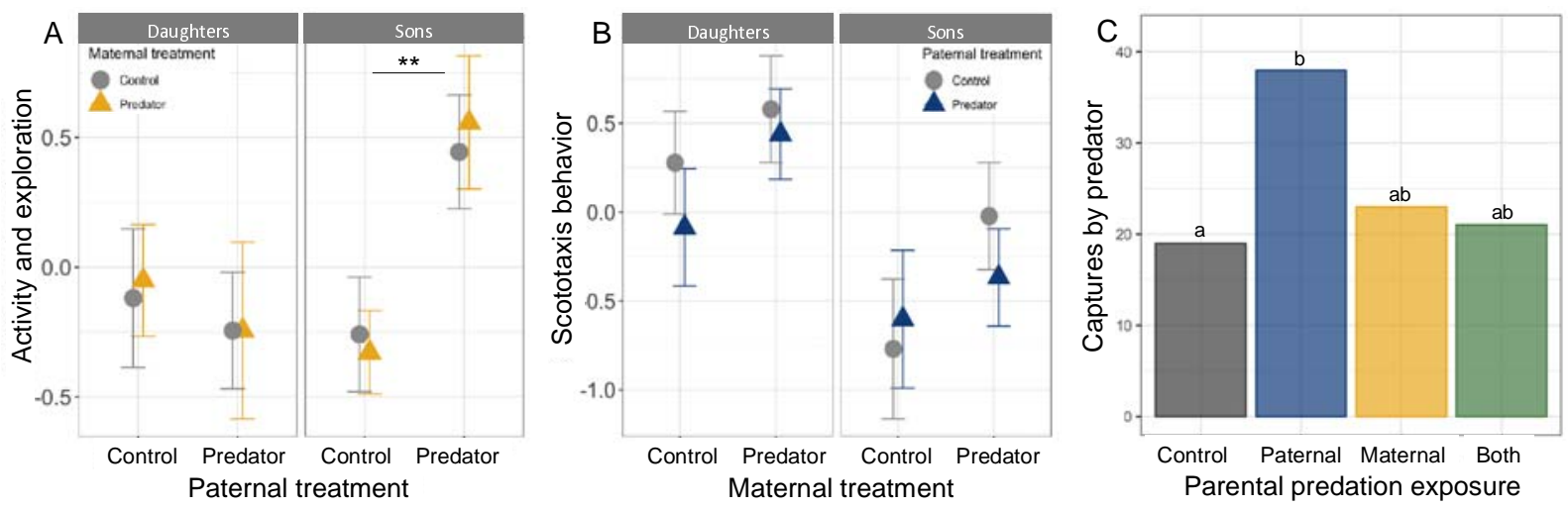

751 Figure 2: The effects of maternal and paternal treatment on offspring in an open field assay,

752 scototaxis assay, and survival in the face of a live predator. A) Male offspring (right) of predator-

753 exposed fathers were significantly more exploratory and active (PCA: higher values indicate

754 more active and exploratory individuals; mean \pm s.e.) compared to male offspring of control

755 fathers; paternal treatment did not affect the exploratory behavior/activity of female offspring

756 (left). The effect of paternal treatment did not depend on maternal treatment (control: grey;

757 predator-exposed: yellow). N=118 offspring. Stars indicate statistically significant differences

758 across treatment groups. B) Offspring of predator-exposed mothers were more cautious (PCA:

759 high values indicate longer latency to enter the white area and spent less time in the white area;

760 mean \pm s.e.) compared to offspring of control mothers. Further, female offspring (left) were more

761 cautious than male offspring (right). The effect of maternal treatment did not depend on paternal

762 treatment (control: grey; predator-exposed: blue). $\mathrm{N}=162$ offspring. C) In live predation trials,

763 juvenile offspring of predator-exposed fathers, but not two predator-exposed parents, were more

764 likely to be captured and consumed by the sculpin predator relative to offspring of control

765 fathers. Shown are the total offspring captured across $n=86$ trials; letters indicate significant

766 differences among treatment groups, determined by Tukey's HSD with parental treatment as a 4-

767 level variable. 

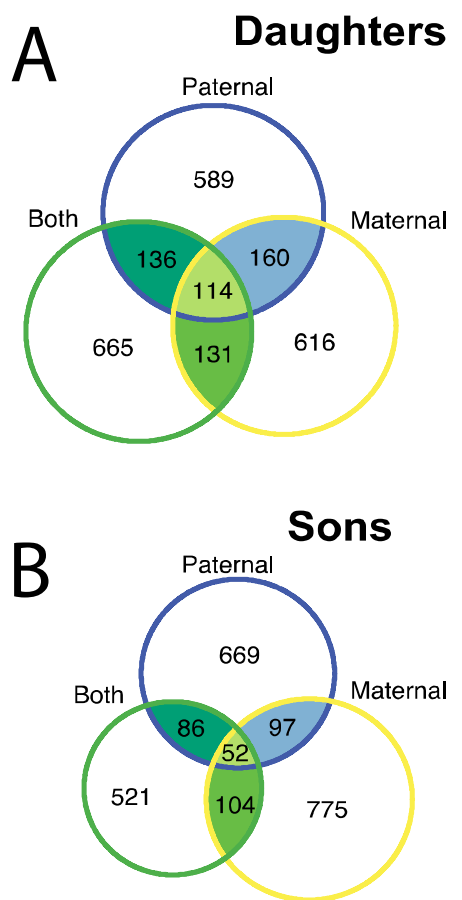
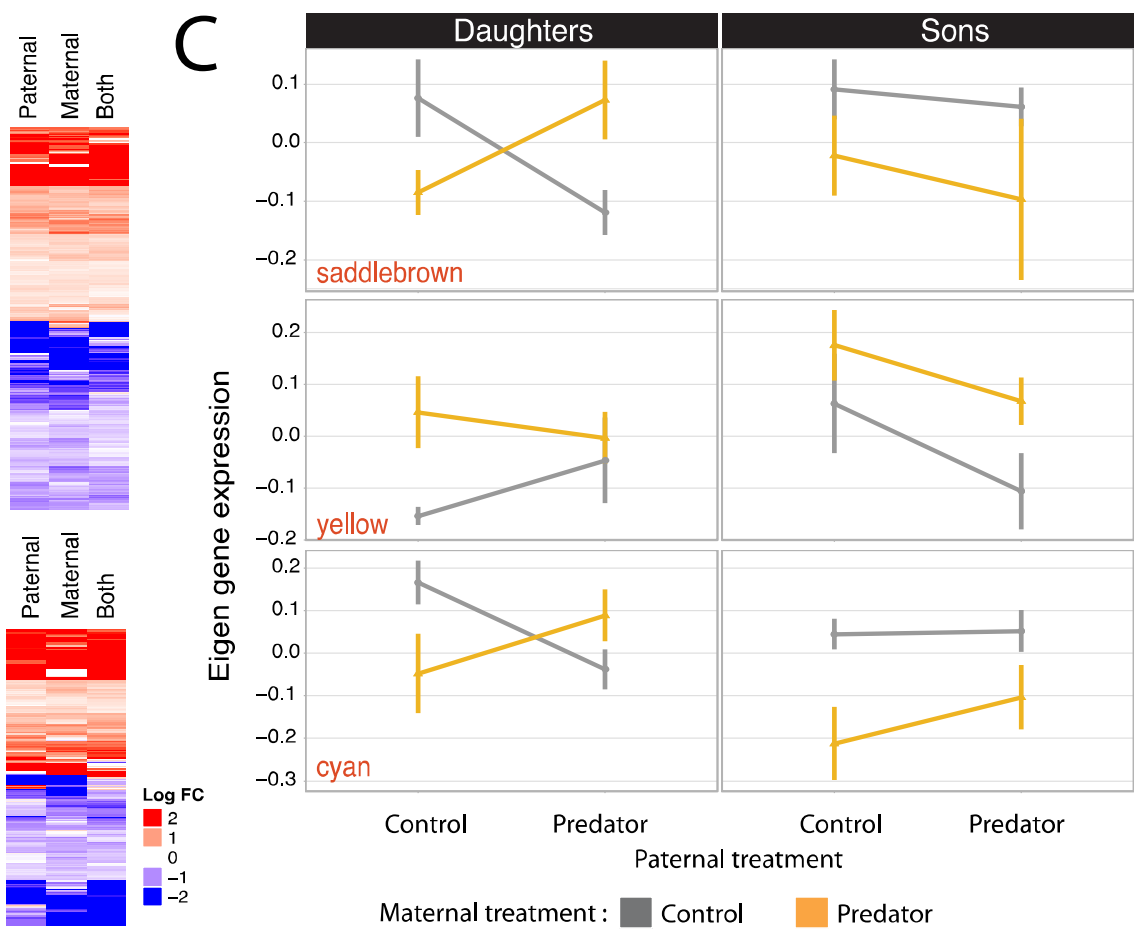

769 Figure 3: Differential gene and eigen-gene expression analysis. A-B) The three circles in the

770 Venn diagram show the number of genes that were differentially expressed in the brain of

771 offspring of unexposed parents relative to offspring of predator-exposed mothers ("maternal"),

772 predator-exposed fathers ("paternal”), or two predator-exposed parents ("both”), with daughters

773 in (A) and sons in (B). Note that relatively few genes overlap between the different pairwise

774 comparisons. The heatmaps show the direction of gene regulation (blue: downregulated; red:

775 upregulated) of the differentially expressed genes that are shared among the three pairwise

776 comparisons, with daughters and sons shown separately. C) The expression profiles of the three

777 eigen-gene modules which were affected by the three-way interaction among paternal treatment,

778 maternal treatment and offspring sex (mean \pm s.e.). $\mathrm{N}=39$ offspring. 